\title{
Utilization of Behavioral Medicine Services to Refine Medical Diagnostic Formulation in the Face of Uncertain Symptom Presentation
}

\author{
David A. Moore ${ }^{a}$ Elisabeth S. Markman ${ }^{a} \quad$ Cori E. McMahon ${ }^{a}$ \\ Maurie Markman ${ }^{b}$ \\ ${ }^{a}$ Division of Behavioral Medicine, Department of Medicine, MD Anderson Cancer Center \\ at Cooper University Hospital, Camden, N.J., USA; ${ }^{b}$ Division of Medicine and Science, \\ Cancer Treatment Centers of America at Eastern Regional Medical Center, \\ Philadelphia, Pa., USA
}

\section{Keywords}

Psycho-oncology · Behavioral medicine - Psychology · Acute promyelocytic leukemia . Endocervical canal cancer · Radiation-induced sarcoma $\cdot$ Interdisciplinary medicine

\begin{abstract}
In the ever expanding realm of cancer care, the psychosocial impact of disease and medical treatments has been garnering increased attention. To address these needs, the integration of behavioral medicine services into inpatient and outpatient medical settings has added a unique resource available to oncologists. Psycho-oncologists may assist providers via the provision of psychological assessment and intervention, supplying valuable consultation to members of the medical team and much needed clinical services to patients. The authors present a complex case in which the utilization of behavioral medicine consultation to clarify the diagnostic picture was critical to identifying underlying anatomic disease.
\end{abstract}

(C) 2016 The Author(s)

Published by S. Karger AG, Basel 


\section{Case Reports in Oncology}

Moore et al.: Utilization of Behavioral Medicine Services to Refine Medical Diagnostic Formulation in the Face of Uncertain Symptom Presentation

\section{Introduction}

The following case elucidates a situation not atypical in psychological practice in a medical setting while exemplifying the critical role of interdisciplinary communication in refining comorbid psychiatric and medical diagnoses and formulating their respective sequelae. The authors present a concise review of salient biopsychosocial information and implications for the incorporation of psychosocial data into the identification of oncologic medical disorders in patients with comorbid psychiatric distress, which may be clouding the diagnostic picture. Prior to discussing the specifics of the case, it is important to provide a foundational understanding of the practice of behavioral medicine in the context of an urban academic cancer center.

Over the course of the past 40 years, psycho-oncology has come of age as its own unique subspecialty in the practice of oncology, vastly refining our knowledge and fostering acceptance of the psychological underpinnings of the cancer experience, which in turn may improve the overall quality of cancer care delivered to the patient [1]. Psychological factors have been shown to be major determinants of individuals' approaches to cancer treatment and thus their medical health outcomes, illuminating a clear link between psychology and physiology which continues to be reinforced in cancer patients [2].

The importance of integrating psychological practice into the comprehensive treatment of cancer patients has become readily apparent, as psychosocial support services are increasing in quantity and breadth. They have been integrated into comprehensive cancer care through consultation-liaison psychiatry, outpatient psychological support services, and most recently through integrative behavioral medicine (IBM) teams at select cancer centers aimed at reducing cancer-related distress and improving patient outcomes. These services offer a tremendous contribution to both patients and interdisciplinary teams. IBM teams in cancer care have demonstrated their direct impact on patient quality of life, treatment regimen adherence, coping with treatment side effects, communication and care between physician and patient, hospital utilization and health outcomes [3-5]. In certain complex cases, such as this one, IBM clinicians also offer a unique perspective on the intricacies of human behavior that may be critical to the identification and delivery of effective oncological treatment.

\section{Case Report}

The patient, a 28-year-old English-speaking Caucasian female, presented for acute inpatient medical hospitalization following complaints of intractable pain originating in her lower back radiating down her left leg. Upon admission, she reported a complex medical history beginning with a diagnosis of acute promyelocytic leukemia at age 15. She was treated with multiagent chemotherapy but experienced a relapse 2 years later. Following additional chemotherapy and a bone marrow transplant, she achieved another remission but relapsed again at age 19 years. This was followed by additional chemotherapy and a second transplant.

In addition, the patient was diagnosed at age 25 years with cervical cancer treated with chemoradiation.

Other notable medical concerns that the patient reported on admission included: a resolved chemotherapy-induced cardiomyopathy (ejection fraction 60\%), documented human papillomavirus infection, and hypothyroidism. There was also a significant familial discord 
with a strong family history of substance abuse in all of her nuclear family members (opiates, cocaine, and alcohol).

In addition to her current concerns related to pain, the patient reported that at the time of admission, she was experiencing increased worry and sadness, subsequently leading to a consultation request for behavioral medicine evaluation to assess for the presence of anxiety and mood disorders, as well as for drug seeking behavior (due to the patient's strong family history of substance abuse).

Upon initial evaluation, the patient reported frustration and worry related to her chronic medical concerns, as well as 'feeling out of control' and 'being tired of being sick all of the time'. She stated that her medical teams had been unable to 'find a reason for [her] pain', and that 'they think it is all in [her] head'. She expanded on her current experiences endorsing: hypersomnolence, increased appetite, significant weight gain, difficulty with concentration ('that may be my chemo brain'), psychomotor retardation, fatigue, and social withdrawal; as well as 'panic attacks two months ago' with the following associated symptoms: shortness of breath, racing heart, diaphoresis, tingling in extremities, and a need to escape the situation.

Throughout the initial evaluation, the patient described consistent anxiety and dysthymic mood, as well as a congruent and reactive affect punctuated by frequent tearfulness. All aspects of her cognition and speech appeared grossly intact, and she evidenced linear and logical thinking, while denying present or past perceptual disturbances or suicidal/homicidal ideation and intent. It is important to note that she stated that she 'was always a positive person' prior to the onset of her new medical concerns.

The patient was assigned a diagnosis of adjustment disorder with mixed anxiety and depressed mood upon ruling out medical etiologies (e.g., hypothyroidism, infection, substance withdrawal/intoxication, electrolyte abnormalities, and others), while noting her recent report of panic attacks, which were in sustained full remission.

Regarding the psychosocial aspects of her care, the patient described that she had recently moved from her country of origin to receive medical care in the United States, and that she was presently separated from all of her support systems, without her nuclear family, with whom there existed considerable relational distress. This lack of social support had compounded her experiences of anxiety and depression as she found herself bereft of her typical coping mechanisms. Specifically, her physical interactions with her peers and romantic partner had typically mitigated prior subclinical psychological distress (i.e., self-reported anxiety and sadness), although due to her separation from them, these interpersonal relationships began to develop a conflicted bidirectional relationship with the patient's psychological distress (e.g., decrease in physical proximity led to increased relational distress with her partner, which then in turn led to increased distress in the patient). Amplifying this distress were increasingly tense relationships with her family, some of whom had relapsed and had begun abusing substances. The patient reported tense relationships with frequent arguments, and that she 'was surprised both of [her] parents were in the same room with each other' as they had experienced a tumultuous divorce during the patient's formative years.

She denied engagement in substance abuse (i.e., tobacco abuse, illicit/licit substance abuse, and alcohol abuse) citing her experiences with family members, although she was presently receiving opiate pain medication to manage her back pain. The patient did not meet criteria for a substance abuse disorder at the time of initial evaluation.

Following discharge, the patient transitioned to the IBM outpatient clinic, where she received ongoing psychological support, although shortly after her initial evaluation, she developed hydronephrosis secondary to ureter scarring from the radiotherapy (received at age 25 years), requiring the placement of bilateral nephrostomy tubes. The patient experienced 


\section{Case Reports in Oncology}

considerable complications related to her nephrostomy tubes, leading to numerous inpatient hospitalizations as a result of infection, ultimately requiring multiple surgical replacements of the nephrostomy tubes over the span of several months.

Subsequent to her concerns related to her nephrostomy tubes, the patient continued to report increased lower back pain with significant radiation throughout her lower left extremity and foot, with new-onset swelling in her left leg and a left foot drop. Upon further examination, the patient was found to have developed a deep venous thrombosis behind her left knee. During this time, the patient evidenced a considerable decrease in mood, paired with decreased functional ability (e.g., difficulty walking with frequent falls, urinary incontinence, decreased ability to engage in activities of daily living), and an increasing need for opiate pain medication.

As a result of her increasing requests for pain medication, the medical teams' concerns shifted to focus more on the possibility of a substance use disorder, with potential malingering, conceptualizing her report of increased pain as a ploy to secure higher doses of intravenous pain medication. Conventional imaging of her lower back had been attempted, although proved unsuccessful due to the presence of significant scaring related to the patient's radiation therapy.

In addition to increased physical concerns, there had been a significant increase in relational distress within the home, which was proposed to have been linked to prolonged hospitalizations as the patient had reported 'I like being in the hospital, I don't have to be at home then'. Additionally, she described 'when I am sleeping I don't have to deal with anything', and was frequently noted to be unarousable in her hospital bed, especially in close temporal proximity to the administration of pain medication.

Despite the utilization of pain medication as a coping mechanism, continuous longitudinal behavioral medicine evaluation across multiple settings reinforced the likelihood of an anatomic etiology for the patient's lower back pain, with recommendations being made to further investigate medical explanations for the pain. Factors which led to this determination included frequent and substantial behavioral observations across settings and at different time points during the treatment process (e.g., before and after surgical intervention, morning vs. evening, prior to and after receiving pain medication, inpatient and outpatient settings, and observation of gait during ambulation to and from clinic rooms), as well as consistency of patient self-report and findings of psychological evaluations over the course of 9 months.

Upon insistence of considering a potential medical etiology, the patient underwent a PET scan which indicated an increase in size and metabolic activity of a previously ruled out left retroperitoneal lesion found to be causing erosive changes in the adjacent left sacrum. Upon further assessment, the patient was assigned a diagnosis of high-grade radiationinduced sarcoma and was scheduled for palliative radiation therapy.

\section{Discussion}

Although a relatively young multidisciplinary subspecialty, the practice of psychooncology has proliferated over the course of the past 4 decades as providers have become more aware of the unique psychosocial changes that oftentimes follow a cancer diagnosis. Due to the multidisciplinary nature of this specialty, psycho-oncologists may practice in a number of settings, including both acute care and outpatient facilities with IBM services. IBM teams are able to meet the unique psychosocial needs of this diverse population by provid- 


\section{Case Reports in Oncology}

Moore et al.: Utilization of Behavioral Medicine Services to Refine Medical Diagnostic Formulation in the Face of Uncertain Symptom Presentation

ing assessment and intervention-based services aimed at assisting with diagnostic clarity and the reduction of physiological/psychological distress and health behavior change (e.g., nonadherence and smoking cessation).

The case at hand illustrates the impactful outcome of ongoing behavioral medicine consultation in both inpatient and outpatient medical settings. Given an unclear medical picture and complex psychosocial factors, initial hypotheses of a substance use disorder and malingering were understandable and essential to assess for. The medical team appropriately consulted behavioral medicine to assess for the presence of psychological distress, and eventually to facilitate effective coping and intervene with behavioral pain management strategies. However, in this particular case, the premature acceptance of these hypotheses as wholly explanatory of a multifaceted presentation precluded the effective identification and treatment of the underlying anatomical issue. Upon reflection on the current case, the authors present a discussion of factors that might, in similar cases, indicate a revisitation of potential medical etiology.

The presence of comorbid physical illness and psychological distress is not an uncommon experience in the realm of oncologic practice, although it may be underreported by patients receiving their routine medical care. When faced with increased stressors, in this case, worsening chronic pain, previous subclinical or clinical psychological distress may become pronounced. In cases where substance abuse and potential malingering occur as primary concerns, this increase in symptom severity may shift the clinical focus and providers' perceptions of patient intentions following the report of symptoms that have dubious anatomical etiologies. Although understandable from a basic communications perspective, these oftentimes brief and difficult interactions between patient and provider may lead to an inadvertent assignment of solely psychological diagnoses that may be masking true physiological distress.

Providers may address these concerns by adopting a multifaceted approach to the assessment of subjective patient problems by conducting sequential evaluations, including behavioral observations as well as potential self-report measures and psychological testing when available. Specifically, assessing the consistency of symptom report, observations of physical functioning in multiple settings (e.g., naturalistic vs. clinical evaluation), and, when appropriate, repeated medical and psychological testing may assist in clarifying diagnostic formulations. Additionally, when available, ongoing IBM evaluation and intervention would provide the medical team with critical insight into relational dynamics and behavioral reinforcement systems that may be influencing the patient's presentation, helping to more accurately conceptualize the impact of psychosocial factors for individual patients.

In closing, it is imperative to approach each case with a gentle curiosity, which allows for diagnostic conceptualizations integrating both psychological and medical factors, rather than viewing distress and dysfunction in these areas as mutually exclusive.

\section{Statement of Ethics}

The authors have no ethical conflicts to disclose.

\section{Disclosure Statement}

The authors have no financial relationships to disclose. 
Moore et al:: Utilization of Behavioral Medicine Services to Refine Medical Diagnostic Formulation in the Face of Uncertain Symptom Presentation

\section{References}

1 Holland JC: History of psycho-oncology: overcoming attitudinal and conceptual barriers. Psychosom Med 2002;64:206-221.

2 Slovic P, Peters E, Finucane M, MacGregor D: Affect, risk, and decision making. Health Psychol 2005;24(suppl 4):S35-S40.

-3 Xia Y, Tong G, Feng R, Chai J, Cheng J, Wang D: Psychosocial and behavioral interventions and cancer patient survival again: hints of an adjusted meta-analysis. Integr Cancer Ther 2014;13:301-309.

-4 Montgomery G, Kangas M, Schnur J, et al: Fatigue during breast cancer radiotherapy: an initial randomized study of cognitive-behavioral therapy plus hypnosis. Health Psychol 2009;28:317-322.

-5 Venetis MK, Robinson JD, Turkiewicz KL, Allen M: An evidence base for patient-centered cancer care: a meta-analysis of studies of observed communication between cancer specialists and their patients. Patient Educ Couns 2009;77:379-383. 